\title{
Estudo de Características do Sistema Mamário e suas Relações com a Produção de Leite em Vacas da Raça Gir ${ }^{1}$
}

\author{
Roberto Luiz Teodoro ${ }^{2}$, Rui da Silva Verneque ${ }^{2}$, Mario Luiz Martinez ${ }^{2}$, Mauro Cruz ${ }^{3}$, Luiz \\ Ronaldo de Oliveira Paula ${ }^{3}$, José de Paula Campos ${ }^{3}$
}

\begin{abstract}
RESUMO - Os objetivos deste trabalho foram estimar as herdabilidades e as correlações genéticas, fenotípicas e de ambiente para produção (T305), altura anterior (ALA) e posterior (ALP) do úbere, comprimento anterior (CAN) e posterior (CPO) das tetas, diâmetro anterior (DAN) e posterior (DPO) das tetas, forma anterior (FA) e posterior (FP) das tetas e forma de ponta de tetas anteriores (FA) e posteriores (FP), por intermédio de um modelo que incluiu os efeitos fixos de ano-estação, rebanho-avaliador, idade à mensuração e efeitos aleatórios de animal (2251 classes), permanente de meio (925 classes) e erro, pelo método de Máxima Verossimilhança Restrita. Produção de leite e características do sistema mamário foram analisadas usando 1804 registros. As estimativas de herdabilidade em análises uni e bivariada foram 0,26 para produção de leite, com variação de 0,09 (FA) a 0,47 (ALA e ALP) para as características do sistema mamário. As estimativas de correlações entre T305, ALA e ALP foram moderadas e negativas $(-0,15$ a $-0,46)$ e menores $(-0,28$ a 0,23$)$ para as demais características do úbere. A seleção para aumento da produção de leite levaria a desejáveis respostas correlacionadas em algumas características do sistema mamário, principalmente altura anterior e posterior do úbere (ALA e ALP).
\end{abstract}

Palavras-chave: correlação genética e fenotípica, herdabilidade, produção de leite, raça Gir, sistema mamário

\section{Study of the Udder System Characteristic and its Relations with Milk Production on Cows from Gyr Breed}

\begin{abstract}
The objectives of this work were to estimate the heritabilities, genetic, phenotypic and environmental correlations for milk production (T305), front (ALA) and rear (ALP) udder height, front (CAN) and rear (CPO) teat length, front (DAN) and rear (DPO) teat diameter, front (FA) and rear (FP) teat shape, front (PA) and rear (PP) teat point shape by means of an animal model that included the fixed effects of year-season, herd-classifier and age at measurement and the random effects of animal (2,251 classes), environmental permanent effect (925 classes) and error by using multiple-trait REML method. Milk production and udder traits were analyzed using 1,804 records. The heritabilities in single and bivariate analysis were 0.26 for T305 and from 0.09 (FA) to 0.47 (ALA and ALP) for the udder system characteristics. The correlations among T305, ALA e ALP were moderate and negative (-0.15 to -0.46) and lower (-0.28 to 0.23$)$ for the other udder characteristics. Selection for improvement of milk production will lead to a favorable correlated response in some udder characteristics, mainly front and rear udder height (ALA and ALP).
\end{abstract}

Key Words: genetic and phenotypic correlation, Gyr breed, heritability, milk production, udder system

\section{Introdução}

Em gado de leite, as características de conformação do úbere assumem grande importância em função de sua associação com características produtivas, o que pode auxiliar na eficiência e redução do tempo de seleção para a produção de leite, por meio da seleção indireta. Poucos estudos têm sido realizados sobre esse assunto; a maioria refere-se às raças européias especializadas, em países de clima temperado (HARRIS et al., 1992; KLASSEN et al., 1992; e KAWAHARA et al., 1996;). A conformação externa do úbere e das tetas da vaca leiteira é importante fator na eficiência da produção. HIGGINS et al. (1980) estudaram, na raça Holandesa, o efeito da herança sobre algumas características de conformação do úbere, assim como suas relações com a saúde do animal. As estimativas de herdabilidade variaram de 0 , para diâmetro médio das tetas, a 0,44 , para comprimento das tetas dianteiras.

BATRA e McALLISTER (1984) estimaram parâmetros genéticos e fenotípicos para medidas de úbere, velocidade de ordenha e produção de leite nas raças Holandesa e Ayrshire, no Canadá. As estima-

\footnotetext{
${ }_{1}$ Projeto atualmente financiado pelo Prodetab e apoio do CNPq.

2 Pesquisador da Embrapa Gado de Leite. Bolsista do CNPq. E-mail: rteodoro@cnpgl.embrapa.br; rsverneque@cnpgl.embrapa.br; martinez@cnpgl.embrapa.br

3 Técnico de campo da ABCGIL e Embrapa Gado de Leite.
} 
tivas de herdabilidade foram moderadas e variaram de 0,22 a 0,61 para medidas de úbere, velocidade de ordenha e produção de leite, em ambas as raças. Vacas de mais alta produção foram ordenhadas em menos tempo e apresentaram úbere mais próximos do solo que as vacas de menor produção. As correlações fenotípicas entre comprimento, diâmetro e distância das tetas com a altura do úbere foram todas negativas, sugerindo que tetas mais longas e mais calibrosas tenderam para úberes mais próximos do solo, concordando com os resultados encontrados por HIGGINS et al. (1980). NORMAN et al. (1988) analisaram as relações entre várias características lineares de tipo e a produção de leite à primeira lactação em cinco raças leiteiras, Ayrshire, Brown Swiss, Guernsey, Jersey e Shorthorn, nos Estados Unidos. De todas as características funcionais para tipo, o caráter leiteiro foi o que apresentou a mais alta correlação fenotípica com produção de leite, em todas as raças estudadas $(0,19$ a 0,53$)$, exceto na Ayrshire, em que a profundidade de úbere $(-0,26)$ e a largura de úbere posterior $(0,21)$ foram superiores. As correlações genéticas entre a produção de leite e o caráter leiteiro variaram de 0,75 e 0,77 nas raças Guernsey e Jersey, respectivamente. As estimativas de herdabilidade variaram de 0,16 a 0,23 para o caráter leiteiro nas cinco raças estudadas e para produção de leite, de 0,17 a 0,31 . HARRIS et al. (1992) obtiveram estimativas de herdabilidade entre 0,12 , para ligamento de úbere anterior, e 0,32 , para comprimento de tetas, estudando as características leiteiras e de úbere na raça Guernsey. Altura e largura de úbere posterior apresentaram a maior correlação genética positiva $(0,85)$, enquanto o tipo leiteiro e a profundidade de úbere apresentaram a maior correlação genética negativa $(-0,41)$. WENCESLAU (1998), analisando dados da raça Gir, obteve estimativas de herdabilidade para as características de úbere, que variaram de 0,07 a 0,46 para diâmetro e comprimento de tetas, respectivamente. As correlações genéticas entre a produção de leite, o comprimento e diâmetro de tetas e a altura do úbere foram $-0,08,-0,12$ e $-0,69$, respectivamente.

As raças zebuínas, destacando-se a Gir, assumem importante papel na pecuária leiteira brasileira, em virtude de sua boa adaptabilidade e do desempenho sob as condições de manejo praticadas no Brasil, tanto como raça pura, como em cruzamentos com raças leiteiras especializadas, principalmente a Holandesa (SANTIAGO, 1975). Portanto, os poucos estudos encontrados na literatura, sobre característi- cas de tipo ou conformação nas raças zebuínas, indicam a necessidade de estudos com essas características e a sua relação com outras economicamente importantes, no sentido de se obter informações para auxiliar a seleção e orientar sistemas de acasalamento para promover o aumento da produtividade animal. O objetivo deste trabalho foi estimar os parâmetros genéticos e fenotípicos entre as principais características do sistema mamário e as de produção em vacas da raça Gir.

\section{Material e Métodos}

Foram utilizadas 1804 informações sobre características do sistema mamário e de produção do leite, de 925 vacas da raça Gir, filhas de 95 touros, com mínimo de três filhas por touro, coletadas no período de 1993 a 1998 em 13 rebanhos que participam do programa de teste de progênie da raça. A idade média das vacas à mensuração foi de $1765 \pm 479$ dias, em diferentes estádios de lactação e ano-época de parto.

Foram medidas 10 características de conformação de úbere e tetas: altura do úbere anterior (ALA) e posterior (ALP), comprimento das tetas anteriores (CAN) e posteriores (CPO), diâmetro das tetas anteriores (DAN) e posteriores (DPO), forma das tetas anteriores (FA) e posteriores (FP), forma das ponta das tetas anteriores (PA) e posteriores (PP), e aferida a produção de leite em até 305 dias de lactação (T305).

As mensurações no sistema mamário foram realizadas em vacas Gir de primeira e segunda lactação, posicionadas em superfícies planas, antes da ordenha, procurando-se fazer no mínimo duas mensurações por animal e por lactação. A altura de úbere posterior e anterior correspondeu à distância entre o solo e as respectivas inserções das tetas anteriores e posteriores. O comprimento das tetas correspondeu à distância entre a ponta da teta e a sua inserção no úbere. O diâmetro das tetas foi medido na porção mediana das mesmas por intermédio de um paquímetro. As tetas foram classificadas quanto à sua forma (1 - cilíndricas, 2 - afuniladas ou 3 - em gargalo) e à forma de suas pontas ( 1 - pontudas, 2 - arredondadas, 3 - achatadas ou 4 - côncavas). A produção de leite em até 305 dias, na primeira e segunda lactação, foram recuperadas do Arquivo Zootécnico do Programa de teste de progênie na raça.

Para estimação dos parâmetros genéticos (herdabilidades, correlações genéticas, fenotípicas e de ambiente), utilizaram-se os componentes de (co) variância obtidos pelo Método da Máxima Verossimi- 
lhança Restrita (REML) usando-se o sistema MTDFREML (BOLDMAN et al., 1995). O modelo utilizado incluiu os efeitos fixos de rebanho-avaliador (64 classes), ano-época de avaliação (29 classes) e a co-variável idade da vaca à avaliação com termos linear e quadrático e os efeitos aleatórios de animal (2251 classes), permanente de meio ( 925 classes) e o erro. A matriz de parentesco incluiu 2251 animais (vaca, pai e mãe).

A natureza categórica das variáveis FA, FP, PA e PP foi ignorada, uma vez que em análise preliminar não houve convergência, ao se utilizar o modelo de limiar (Threshold - model), proposto por MISZTAL (1989).

\section{Resultados e Discussão}

Médias, desvio-padrão e valores máximos e mínimos para as características do sistema mamário e de produção são apresentados na Tabela 1. Observa-se, nesta Tabela, que há pequena diferença entre ALA e ALP, com tendência de a porção anterior do úbere ser mais elevada que a porção posterior. Para altura de úbere, em relação ao piso, as médias encontradas neste estudo foram maiores que as encontradas por HIGGINS et al. (1980), os quais observaram média de 54,6 $\pm 6,8 \mathrm{~cm}$ para altura do úbere em vacas da raça Holandesa, e por BATRA e McALLISTER (1984), cujas médias foram 54,9 $\pm 4,0 \mathrm{~cm}$ e 51,6 $\pm 4,4 \mathrm{~cm}$ para as raças Holandesa e Ayrshire, respectivamente.
Esta diferença possivelmente é atribuída à maior capacidade de produção das raças européias especializadas que apresentam, conseqüentemente, maior volume glandular em relação às raças nãoespecializadas, como o Zebu, neste caso. Para o comprimento e diâmetro de tetas, as médias encontradas neste estudo foram também diferentes das encontradas por ambos os autores, que observaram médias bem inferiores às da raça Gir, o que seria mais vantajoso, evidenciando possível seleção para adequação ao manejo de ordenha destas raças especializadas. Em ambos os estudos, as tetas anteriores também foram mais longas e mais calibrosas que as posteriores, como ocorreu neste estudo.

As estimativas dos componentes de variância genética aditiva e residual, obtidas em uma análise univariada, assim como as herdabilidades para as características estudadas, são apresentadas na Tabela 2.

$\mathrm{Na}$ Tabela 3, são apresentadas as estimativas das herdabilidades, correlações genéticas, fenotípicas e ambientes entre a produção de leite e as características do sistema mamário, obtidas de análises bivariadas. As estimativas de herdabilidade para altura de úbere foram constantes $(0,47)$ e, para comprimento de tetas, variaram de moderadas a altas $(0,38$ a 0,42$)$, nas análises uni e bivariada. Para diâmetro $(0,18$ a 0,24$)$ e forma das tetas $(0,09$ a 0,11$)$ e forma de ponta $(0,17$ a 0,18$)$, as estimativas foram de moderadas a baixas, concordando com os resultados

Tabela 1 - Estatísticas descritivas para as características do sistema mamário e de produção de leite de vacas da raça Gir

Table 1 - Descriptive statistics for udder system and milk production characteristics of Gyr cow breed

\begin{tabular}{lcccc} 
Característica & $\begin{array}{c}\text { Média }(\mathrm{cm}) \\
\text { Tean }\end{array}$ & $\begin{array}{c}\text { Desvio-padrão }(\mathrm{cm}) \\
\text { Standard deviation }\end{array}$ & \multicolumn{2}{c}{$\begin{array}{c}\text { Amplitude }(\mathrm{cm}) \\
\text { Range }\end{array}$} \\
\cline { 4 - 5 } & & & $\begin{array}{c}\text { Mínima } \\
\text { Minimum }\end{array}$ & $\begin{array}{c}\text { Máxima } \\
\text { Maximum }\end{array}$ \\
\hline ALA & 61,35 & 4,70 & 45,0 & 75,0 \\
ALP & 60,39 & 4,49 & 43,0 & 73,0 \\
CAN & 7,89 & 1,95 & 3,0 & 15,0 \\
CPO & 7,09 & 1,84 & 2,0 & 14,2 \\
DAN & 3,69 & 0,69 & 1,5 & 7,0 \\
DPO & 3,32 & 0,61 & 1,0 & 5,5 \\
FA & 1,77 & 0,79 & 1,0 & 3,0 \\
FP $^{1}$ & 1,58 & 0,64 & 1,0 & 3,0 \\
PA $^{2}$ & 1,61 & 0,65 & 1,0 & 4,0 \\
PP $^{2}$ & 1,59 & 0,66 & 1,0 & 4,0 \\
T305 $^{3}$ & $2.777,55$ & 993,05 & 438,0 & 7053,0
\end{tabular}

$1=$ classes de 1 a $3 ; 2$ = classes de 1 a $4 ; 3=\mathrm{kg}$.

1 = classes from 1 to $3 ; 2$ = classes from 1 to $4 ; 3=\mathrm{kg}$.

ALA e ALP = altura anterior e posterior do úbere $(A L A$ and $A L P=$ front and rear udder height); $C A N$ e $C P O=$ comprimento das tetas anteriores e posteriores (CAN and CPO = front and rear teat length); DAN e DPO = diâmetro das tetas anteriores e posteriores ( $D A N$ and $D P O=$ front and rearteat diameter); $F A$ e FP $=$ forma das tetas anteriores e posteriores (front and rear teat shape); $\mathrm{PA}$ e PP = forma das pontas das tetas anteriores e posteriores $(P A$ and $P P=$ front and rear teat point shape); T305 = produção de leite até 305 dias de lactação (T305 = milk production). 
134

Tabela 2 - Estimativas de componentes de variância genética aditiva $\left(\hat{\sigma}_{\mathrm{a}}^{2}\right)$ e residual $\left(\hat{\sigma}_{e}^{2}\right)$ e de herdabilidades $\left(\hat{\mathrm{h}}^{2}\right)$, em análise univariada

Table 2 - Estimates of genetic additive $\left(\hat{\sigma}_{a}^{2}\right)$ and residual $\left(\hat{\sigma}_{e}^{2}\right)$ variance components and heritabilities $\left(\hat{\mathrm{h}}^{2}\right)$ from single-trait analysis

\begin{tabular}{llll}
\hline Característica & $\hat{\sigma}_{\mathrm{a}}^{2}$ & $\hat{\sigma}_{e}^{2}$ & $\hat{\mathrm{h}}^{2}$
\end{tabular}

\section{Trait}

\begin{tabular}{lccc}
\hline ALA & 6,45 & 3,09 & $0,47 \pm 0,09$ \\
ALP & 6,15 & 2,89 & $0,47 \pm 0,09$ \\
CAN & 1,02 & 0,84 & $0,38 \pm 0,08$ \\
CPO & 0,94 & 0,81 & $0,42 \pm 0,08$ \\
DAN & 0,05 & 0,13 & $0,18 \pm 0,06$ \\
DPO & 0,06 & 0,12 & $0,24 \pm 0,07$ \\
FA & 0,05 & 0,38 & $0,09 \pm 0,05$ \\
FP & 0,04 & 0,27 & $0,11 \pm 0,05$ \\
PA & 0,07 & 0,26 & $0,18 \pm 0,06$ \\
PP & 0,07 & 0,25 & $0,17 \pm 0,06$ \\
T305 & $146.082,54$ & $137.161,32$ & $0,26 \pm 0,08$ \\
\hline
\end{tabular}

$\mathrm{ALA}$ e ALP = altura anterior e posterior do úbere ( $A L A$ and $A L P=$ front and rear udder height); CAN e CPO = comprimento das tetas anteriores e posteriores (CAN and CPO = front and rear teat length); DAN e DPO = diâmetro das tetas anteriores e posteriores $(D A N$ and $D P O=$ front and rear teat diameter) $; \mathrm{FA}$ e FP = forma das tetas anteriores e posteriores (front and rear teat shape); PA e PP = forma das pontas das tetas anteriores e posteriores $(P A$ and $P P=$ front and rear teat point shape); T305 = produção de leite até 305 dias de lactação $($ T305 = milk production up to 305 days of lactation). obtidos por HIGGINS et al. (1980), BATRA e McALLISTER (1984) e WENCESLAU (1988). Características do sistema mamário, com estimativas de herdabilidades moderadas, indicam que é possível se obter ganho genético nas mesmas por intermédio da seleção. O comprimento de tetas aparenta ser mais herdável que o diâmetro, indicando que a seleção para esta característica pode ser mais efetiva. Produção de leite $(0,26)$ também apresenta potencial para melhoramento por meio da seleção, principalmente pelo teste de progênie.

As correlações genéticas, fenotípicas e ambientes entre altura de úbere anterior e posterior com a produção de leite foram negativas e moderadas, como observado na Tabela 3 , indicando que, quanto mais alto o úbere, ou seja, quanto menor a sua capacidade glandular, menor é a produção de leite. A seleção para a produção de leite possivelmente proporcionaria como resposta correlacionada maior volume glandular mamário e, conseqüentemente, úberes mais baixos, concordando com os resultados de

Tabela 3 - Estimativas de herdabilidades $\left(\hat{\mathrm{h}}^{2}\right)$, correlações genéticas $\left(\hat{\mathrm{r}}_{\mathrm{g}}\right)$, fenotípicas $\left(\hat{\mathrm{r}}_{\mathrm{p}}\right)$ e ambientes $\left(\hat{\mathrm{r}}_{\mathrm{e}}\right)$ entre a produção de leite (T305) e as características do sistema mamário, em análises bivariadas

Table 3 - Estimates of heritabilities $\left(\hat{\mathrm{h}}^{2}\right)$, genetic $\left(\hat{\mathrm{r}}_{\mathrm{g}}\right)$, phenotypic $\left(\hat{\mathrm{r}}_{\mathrm{p}}\right)$ and environmental $\left(\hat{\mathrm{r}}_{\mathrm{e}}\right)$ correlations between milk production (T305) and udder traits, from bivariate analysis

\begin{tabular}{lcccc}
\hline $\begin{array}{l}\text { Característica } \\
\text { Trait }\end{array}$ & $\hat{\mathrm{h}}^{2}$ & $\hat{\mathrm{r}}_{\mathrm{g}}$ & $\hat{\mathrm{r}}_{\mathrm{p}}$ & $\hat{\mathrm{r}}_{\mathrm{e}}$ \\
\hline ALA & & & & \\
ALP & 0,47 & $-0,46$ & $-0,25$ & $-0,15$ \\
CAN & 0,47 & $-0,44$ & $-0,26$ & $-0,17$ \\
CPO & 0,41 & $-0,28$ & 0,08 & 0,03 \\
DAN & 0,42 & $-0,09$ & 0,13 & 0,05 \\
DPO & 0,18 & $-0,07$ & 0,19 & 0,03 \\
FA & 0,24 & $-0,08$ & 0,17 & 0,07 \\
FP & 0,09 & 0,00 & 0,02 & $-0,04$ \\
PA & 0,11 & $-0,03$ & 0,03 & $-0,09$ \\
PP & 0,18 & 0,23 & 0,01 & 0,08 \\
ALA ALP & 0,17 & 0,03 & 0,00 & 0,09 \\
\hline
\end{tabular}

ALA e ALP = altura anterior e posterior do úbere $(A L A$ and $A L P=$ front and rear udder height $)$; $\mathrm{CAN}$ e $\mathrm{CPO}=$ comprimento das tetas anteriores e posteriores $(C A N$ and $C P O=$ front and rear teat length); DAN e DPO = diâmetro das tetas anteriores e posteriores $(D A N$ and DPO = front and rear teat diameter) ; FA e FP = forma das tetas anteriores e posteriores (front and rear teat shape); $\mathrm{PA}$ e PP = forma das pontas das tetas anteriores e posteriores $(P A$ and $P P=$ front and rear teat point shape); T305 = produção de leite até 305 dias de lactação $(T 305=$ milk production up to 305 days of lactation). 
MISZTAL et al. (1992) na raça Holandesa.

As correlações genéticas, fenotípicas e ambientes entre a produção de leite e as características das tetas foram todas relativamente baixas, variando de $-0,28$ a +0,23 nas correlações genéticas, 0 a 0,19 nas fenotípicas e -0,09 a +0,09 nas ambientes. Em virtude de existirem poucos trabalhos sobre o assunto, principalmente nas raças zebuínas, deve-se elaborar estudos para avaliação do potencial produtivo dessas raças e obtenção de maiores informações. Para maior precisão nos resultados, torna-se necessário, portanto, maior número de informações, requerendo-se maior número de animais e de mensurações por animal.

Estimativas de herdabilidade para características do sistema mamário e produção de leite, obtidas neste estudo, variaram de baixas a moderadas.

\section{Conclusões}

As correlações entre produção de leite e as características do sistema mamário foram baixas, exceto para altura do úbere, que foram moderadas, evidenciando nesta população independência genética entre a produção e as características de úbere.

\section{Agradecimentos}

Ao CNPq e aos criadores de gado Gir, por intermédio da Associação Brasileira de Criadores de Gir Leiteiro (ABCGIL), pelas bolsas de estudo e pelo apoio financeiro e logístico na realização deste trabalho.

\section{Referências Bibliográficas}

BATRA, T.R., McALLISTER, A.J. 1984. Relationships among udder measurements, milking speed, milk yield, and CMT scores in young dairy cows. Can. J. Anim. Sci., 64: 807-815.

BOLDMAN, K.G., KRIESE, L.A., VAN VLECK, L.D. et al. 1995. A manual for use of MTDFREML. A set of programs to obtain estimate of variances and covariances [DRAFT]. Lincoln: USDA/ARS. 120p.
HARRIS, B.L., FREEMAN, A.E., METZGER, E. 1992. Genetic and phenotypic parameters for type and production in Guernsey dary cows. J. Dairy Sci., 75(4):1147-1153.

HIGGINS, S., MOORE, R.K., KENNEDY, B.W. 1980. Heritabilities of teat conformation traits and their relationships with somatic cell count in Holsteins. Can. J. Anim. Sci., 60:231-239.

KAWAHARA, T., SUZUKI, M., IKEUCHI, Y. 1996. Genetic parameters of production and type traits and longevity in Holstein population. Anim. Sci. and Tech., 67(5):463-475.

KLASSEN, D.J., MONARDES, H.G., JAIRATH, L. et al. 1992. Genetic correlations between lifetime production and linearized type in Canadian Holsteins. J. Dairy Sci., 75(8):2272-2282.

MISZTAL, I., GIANOLA, D., FOULEY, J.L. 1989. Computing aspects of nonlinear method of sire evaluation for categorical data. J. Dairy Sci., 53:643-650.

MISZTAL, I., LAWLOR, T.J., SHORT, T.H. et al. 1992. Multiple-trait estimation of variance components of yield traits using an animal model. J. Dairy Sci., 75(2):544-551.

NORMAN, H.D., POWELL, R.L., WRIGHT, J.R. et al. 1988. Phenotypic and genetic relationship between linear functional type traits and milk yield for five breeds. J. Dairy Sci., 71:1880-1888.

SANTIAGO, A.A. 1975. Os cruzamentos na pecuária bovina. São Paulo: Instituto de Zootecnia. 549p.

WENCESLAU, A.A. 1998. Estudo de medidas de conformação e suas relações com características produtivas e reprodutivas em vacas da raça Gir Leiteiro. Viçosa, MG: UFV, 1998. 75p. Dissertação (Mestrado em Melhoramento Animal), Universidade Federal de Viçosa, 1988.

Recebido em: 08/09/98

Aceito em: 09/07/99 